\title{
Different Impacts of Luminosity on Contrast Vision in Eyes with Transparent Optic Media and with Cataract Simulation
}

\author{
Adrian Smedowski ${ }^{a-c}$ Karolina Mikus-Zagorska ${ }^{a} \quad$ Agnieszka Jakubowska $^{a}$ \\ Anna Piotrowska-Gwozdz ${ }^{a} \quad$ Agnieszka Piotrowska-Seweryn $^{a}$ Patrycja Orchel $^{a}$ \\ Dorota Pojda-Wilczek ${ }^{a}$ \\ ${ }^{a}$ Ophthalmology Clinic and Department of Ophthalmology, 5th Clinical Hospital, and bepartment of Physiology, \\ School of Medicine in Katowice, and ${ }^{C}$ Department of Ophthalmology, School of Medicine and Division of Dentistry \\ in Zabrze, Medical University of Silesia, Katowice, Poland
}

\section{Key Words}

Contrast vision · Cataract · Myopia · Luminosity

\begin{abstract}
Objective: To evaluate the effect of luminous intensity on contrast vision under different ocular conditions. Materials and Methods: Ninety eyes of 45 persons were included in this study as follows: 30 healthy eyes, 30 eyes with cataract simulation (using translucent glasses), and 30 myopic eyes. Contrast sensitivity was examined using 5 spatial frequencies $(1.5,3.0,6.0,12.0$, and 18.0 cycles per degree) of sine wave contrast test optotypes for 4 light intensities $(34,68$, 154 , and $240 \mathrm{~cd} / \mathrm{m}^{2}$ ). Results: The mean linear contrast sensitivities averaged over the frequencies for each of the 4 light intensities were: healthy eyes: $59 \pm 11,72 \pm 16,79 \pm 23$, and $80 \pm 19$; myopic eyes: $52 \pm 13,67 \pm 15,73 \pm 21$, and $75 \pm 18$, and cataract simulation eyes: $15 \pm 7,21 \pm 8.6,28.7 \pm 13$, and $28.6 \pm 13$, respectively. The linear contrast sensitivities averaged over the light intensities for each of the 5 spatial frequencies were: healthy eyes: $78,87,117,59$, and 21 ; myopic eyes: $65,84,109,54$, and 29 , and cataract simulation eyes: 37 , 41, 28, 8, and 2. Conclusions: The light intensity level had a positive effect on the contrast sensitivity of the examined
\end{abstract}

\begin{tabular}{ll}
\hline KARGER 125/\% & $\begin{array}{l}\text { (2) 2015 S. Karger AG, Basel } \\
1011-7571 / 15 / 0246-0501 \$ 39.50 / 0 \quad \text { Karger }\end{array}$ \\
E-Mail karger@karger.com & $\begin{array}{l}\text { This is an Open Access article licensed under the terms of the } \\
\text { www.karger.com/mpp }\end{array}$ \\
$\begin{array}{l}\text { Creative Commons Attribution-NonCommercial 3.0 Un- } \\
\text { ported license (CC BY-NC) (www.karger.com/OA-license), } \\
\text { applicable to the online version of the article only. Distribu- } \\
\text { tion permitted for non-commercial purposes only. }\end{array}$
\end{tabular}

eyes, except for eyes with cataract simulation, where even the maximum light intensity did not improve the contrast vision. This indicates that patients with cataracts require increased contrast of text rather than brighter illumination to improve the quality of their vision.

(c) 2015 S. Karger AG, Basel

\section{Introduction}

Contrast is a physical variable that can be defined as the specific difference between the luminosities (luminous intensities) of 2 adjacent points [1]. Contrast vision is described as the ability of the eye to distinguish and interpret different luminosity areas as separate points. Contrast sensitivity is the smallest possible difference in object luminosity that can be differentiated by the eye $[2,3]$. Luminosity is used to describe the amount of light reflected from the surface and perceived by the eye [4].

Contrast sensitivity is a function of the inner retinal layers that depends directly on the cones [5]. Functionally, contrast vision is related to stimulation of 'on-off receptive fields belonging to retinal ganglion cells. The location and size of these fields determine the light sensi-
Dorota Pojda-Wilczek, MD, PhD

Ophthalmology Clinic and Department of Ophthalmology

5 th Clinical Hospital, Medical University of Silesia, Ceglana 35

PL-40-952 Katowice (Poland)

E-Mail pojda-wilczek@wp.pl 
tivity and contrast resolution of vision [6]. A small, isolated receptive field, where a single retinal ganglion cell is connected with a single photoreceptor, is characterized by high-contrast resolution but a low sensitivity to stimuli, as observed in the central retina $[6,7]$. The system of big receptive fields, where a single retinal ganglion cell is connected with many photoreceptors, and the receptive fields of neighboring retinal ganglion cells overlap, is characterized by low-contrast resolution with a high sensitivity to stimulus and is present mostly in the peripheral retina [6,7]. Contrast sensitivity is the second variable, after visual acuity, that describes the minimal resolution ability of the eye. Visual acuity measures the eye's spatial resolution for highly contrasted objects, while contrast sensitivity measures the minimum luminosity difference between these objects $[1,8]$. The state of contrast vision depends on retinal neuron function as well as on the eye's optic media transparency. Decreased contrast sensitivity can be a sign of pathologies that involve the retina or the optic media of the eye, such as amblyopia, cataract, glaucoma, or diabetic retinopathy [9-13]. Reduced contrast sensitivity can also appear as a side effect of corneal refractive surgery procedures [14]. It has been reported that the evaluation of contrast sensitivity might serve as a screening procedure for glaucoma [10]. Furthermore, some neuroophthalmological diseases that do not influence visual acuity directly can be detected by examining retinal contrast sensitivity, such as in sclerosis multiplex or some neoplasms [15].

In this study, we evaluated the influence of luminosity on contrast sensitivity in healthy and myopic eyes as well as in eyes with cataract simulation.

\section{Materials and Methods}

This study was approved by the Institutional Ethical Scientific Committee and based on the Declaration of Helsinki. Written informed consent was obtained from each participant after explaining the purpose of this study.

\section{Study Groups}

The study was conducted on 90 eyes (45 persons, age range 23-26 years) divided into 3 groups ( 15 persons each) as follows: 30 healthy eyes, 30 myopic eyes (refractive error ranged between -1.0 and $-7.0 \mathrm{D}$ ), and 30 eyes with cataract simulation (with translucent glasses). The inclusion criteria were: for the healthy group, a spherical refractive error between -1.0 and $+1.0 \mathrm{D}$, astigmatism of less than $0.5 \mathrm{D}$, decimal visual acuity without correction, $\mathrm{V}=$ 1.0 (based on the 5-meter Snellen chart) and $\mathrm{Sn}=0.5$ (based on the 0.5-meter Snellen chart); for the cataract group, a spherical refractive error between -1.0 and $+1.0 \mathrm{D}$, astigmatism of less than
$0.5 \mathrm{D}$, decimal visual acuity without correction, $\mathrm{V}=1.0$ (without translucent glasses, based on the 5-meter Snellen chart) and $\mathrm{Sn}=$ 0.5 (with and without translucent glasses, based on the 0.5 -meter Snellen chart), and for the myopia group, a spherical refractive error greater than $-1.0 \mathrm{D}$, astigmatism of less than $0.5 \mathrm{D}$, best corrected decimal visual acuity, V = 1.0 (based on the 5-meter Snellen chart) and $S n=0.5$ (based on the 0.5 -meter Snellen chart). To reduce possible bias and deviations within groups, this study was conducted on groups of matched age, visual acuity, and optic media transparency. To achieve this last goal, we introduced uniform cataract simulation with translucent glasses instead of recruiting cataract patients, since lens opacity in cataracts and the accompanying changes in the eye, related to the age of the cataract patient, tend to vary.

\section{Materials}

This study was based on the sine wave contrast test using a VCTS 6000 (Stereo Optical, Dayton, Ohio, USA). The test consisted of 45 sine wave patches ordered in 5 rows and 8 columns. In rows A-E, optotypes were sorted by increasing spatial frequency $[1.5,3.0,6.0,12.0$, and 18.0 cycles per degree (cpd)] into columns numbered $1-8$ by decreasing contrast. The test chart was illuminated using a light source of 1 out of 4 different intensities corresponding to fixed chart luminosities (1-4): 34, 68, 154, and $240 \mathrm{~cd} /$ $\mathrm{m}^{2}$, set with the light meter (Stereo Optical). Cataract was simulated by using diffusing translucent glasses of equal transparency (Stereo Optical) (fig. 1).

\section{Study Design}

The study participants were first examined for noncorrected (healthy and cataract groups) or best corrected (myopia group) 5and 0.5 -meter visual acuity and the tests were performed in a welllit room. Participants were examined next using the sine wave contrast test of increasing light intensity after $10 \mathrm{~min}$ of dark adaptation, separately for each eye, for a fixed distance from the test of 46 $\mathrm{cm}$. After measurements, values of contrast sensitivity were decoded based on the key and expressed as linear contrast sensitivity (fig. 2).

\section{Statistical Analysis}

A statistical analysis was performed using IBM SPSS Statistics 20 (IBM, Armonk, N.Y., USA) and MedCalc 12.3.0 (MedCalc Software, Ostend, Belgium). Descriptive statistical results are presented as means \pm SD. The Kolmogorov-Smirnov test was used to evaluate whether the data were normally distributed. Data were compared using the paired-samples Wilcoxon test, the Kruskal-Wallis test, or the Mann-Whitney U test. $\mathrm{p}<0.05$ was considered statistically significant.

\section{Results}

\section{Differences between Groups}

Compared to other groups, eyes with cataract simulation had up to 4 times lower mean values of contrast sensitivity (ranging between $15.7 \pm 7$ and $28.8 \pm 13$, when for healthy and myopic eyes they ranged from $59.4 \pm 11$ to $80.3 \pm 19$ and from $52.1 \pm 13$ to $75.3 \pm 18$, respectively) 
Fig. 1. Sine wave contrast test with additional elements. a Sine wave contrast test chart consisting of 45 sine wave patches of known spatial frequency ordered in 8 columns and 5 rows. In rows A-E, optotypes are sorted by increasing spatial frequency in columns numbered $1-8$ by decreasing contrast. b Light meter used to set the luminosity values: $34,68,154$, and $240 \mathrm{~cd} / \mathrm{m}^{2}$. The green area corresponds to the optimal luminous intensity range of $68-240 \mathrm{~cd} / \mathrm{m}^{2}$ (color refers to the online version only). Sample presentation of 3 different luminosities measured with the light meter. c Cataract simulation glasses. Sine wave contrast test chart without (d) and with (e) cataract simulation glasses.
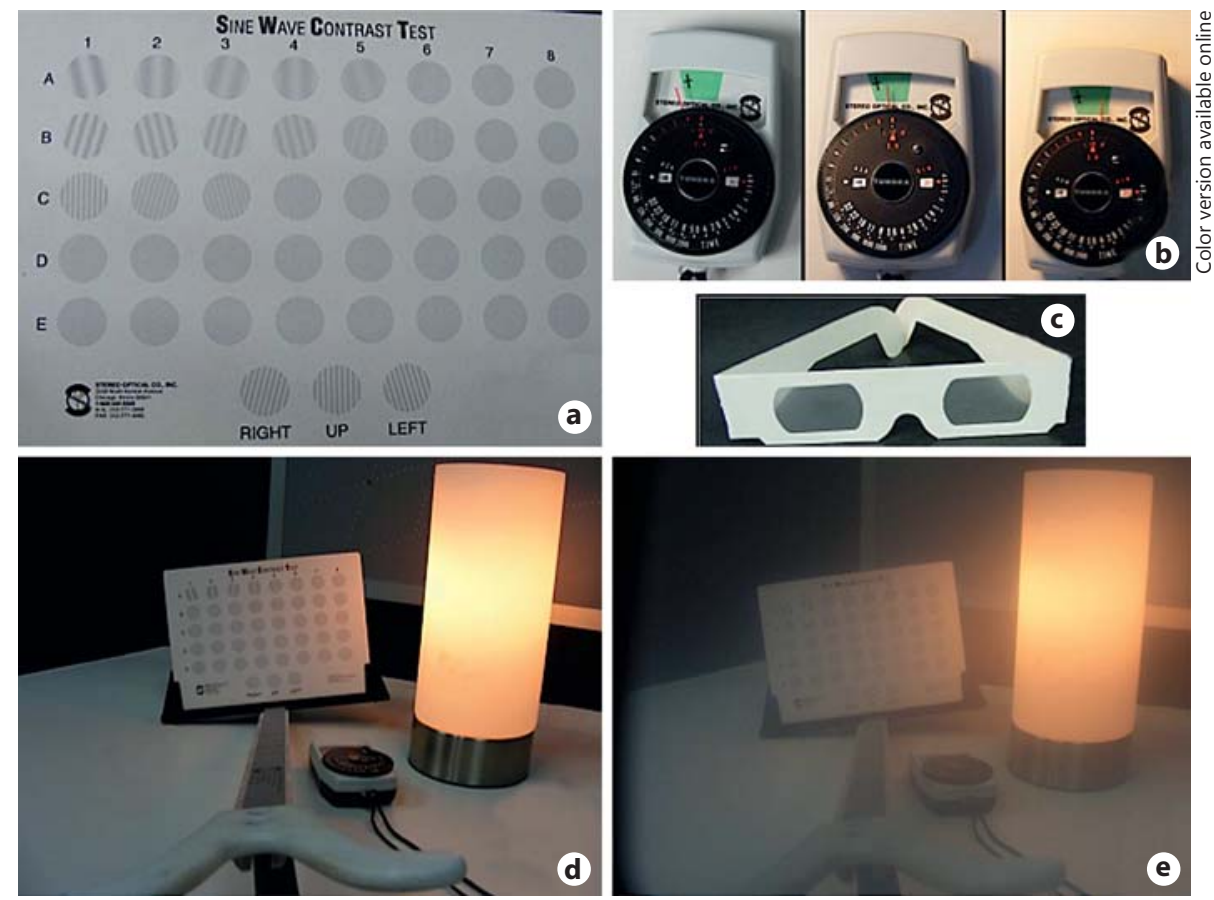

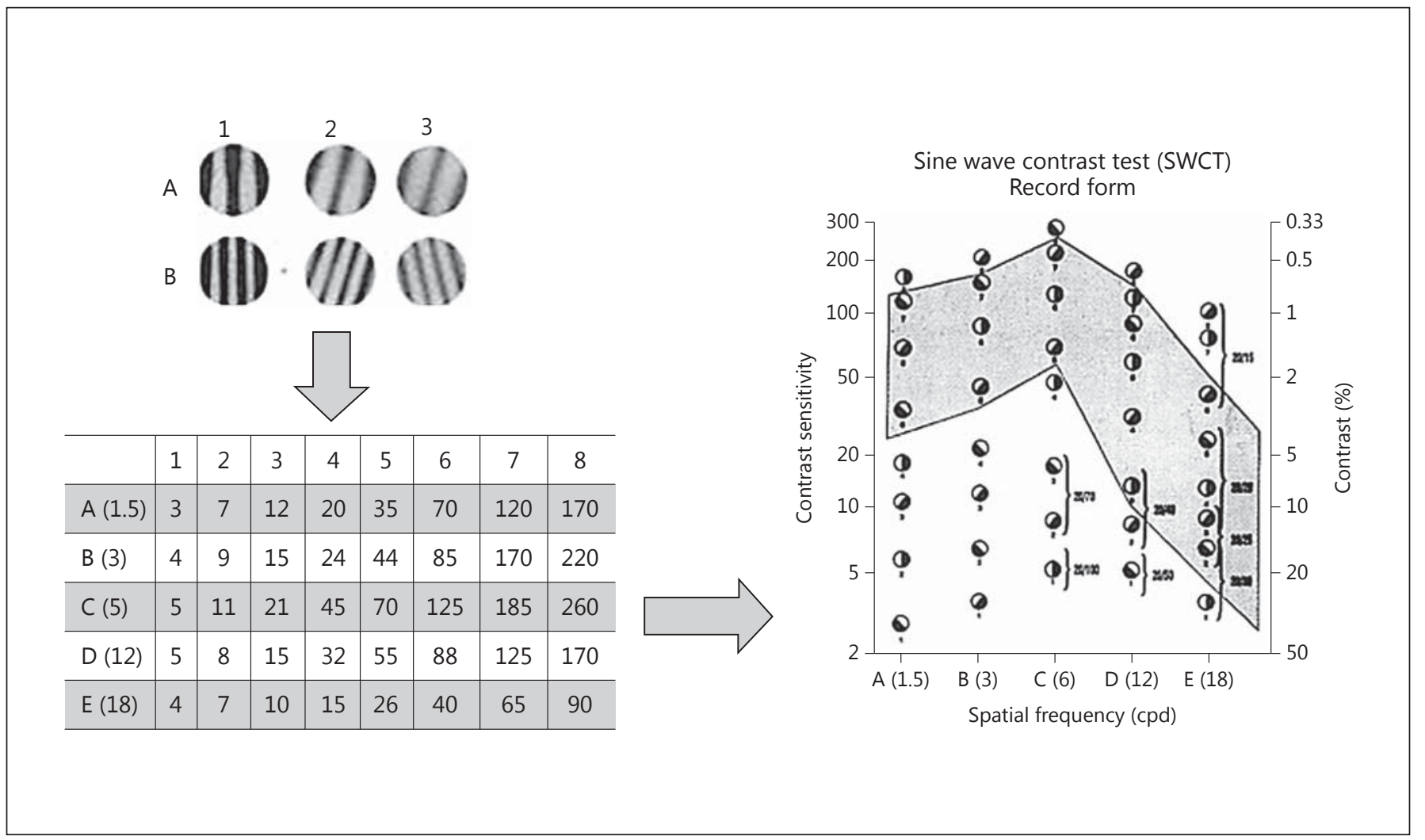

Fig. 2. Linear contrast sensitivity value interpretation. The values were decoded based on the key and marked on a contrast vision record form. 


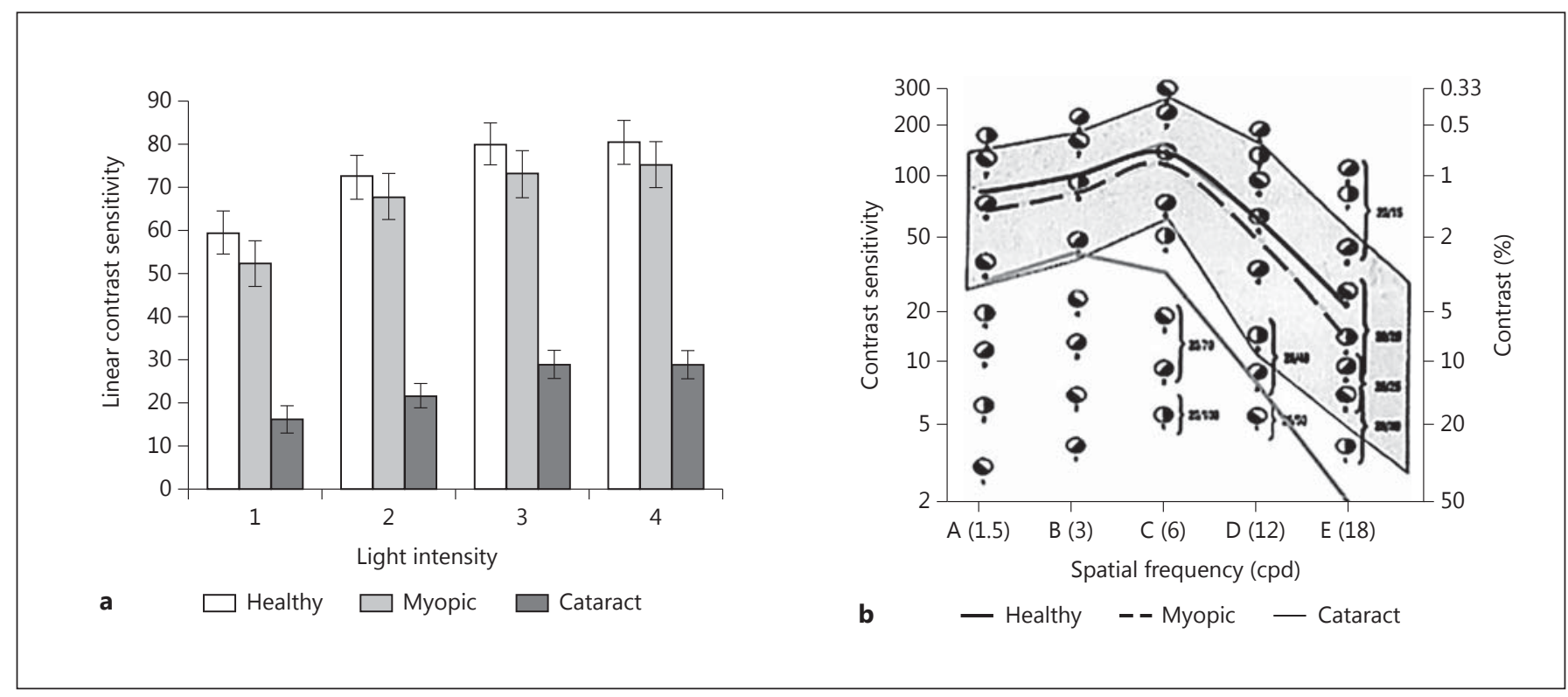

Fig. 3. a Mean linear contrast sensitivities for each light intensity in healthy, myopic, and cataract eyes. b Contrast vision record form: mean linear contrast sensitivities for each spatial frequency in healthy, myopic, and cataract eyes.

Table 1. Linear contrast sensitivity for emmetropic, myopic, and cataract eyes

\begin{tabular}{|c|c|c|c|c|c|c|}
\hline & A & $\mathrm{B}$ & $\mathrm{C}$ & $\mathrm{D}$ & $\mathrm{E}$ & Mean \\
\hline Lower reference limit of contrast sensitivity ${ }^{a}$ & 35.0 & 44.0 & 70.0 & 15.0 & 7.0 & 34.2 \\
\hline Healthy eyes (luminosity 1 ) & $80.8 \pm 24$ & $87.8 \pm 12$ & $91.3 \pm 21$ & $29.4 \pm 6$ & $7.9 \pm 2$ & $59.4 \pm 11$ \\
\hline Healthy eyes (luminosity 2 ) & $76.3 \pm 22$ & $85.0 \pm 11$ & $123.2 \pm 26$ & $57.7 \pm 17$ & $19.3 \pm 5$ & $72.3 \pm 16$ \\
\hline Healthy eyes (luminosity 3 ) & $81.0 \pm 19$ & $90.7 \pm 28$ & $126.1 \pm 33$ & $74.6 \pm 21$ & $27.6 \pm 13$ & $80.0 \pm 23$ \\
\hline Healthy eyes (luminosity 4) & $74.2 \pm 16$ & $87.9 \pm 14$ & $129.0 \pm 41$ & $77.0 \pm 20$ & $33.6 \pm 6.5$ & $80.3 \pm 19$ \\
\hline Myopic eyes (luminosity 1 ) & $56.5 \pm 14$ & $80.9 \pm 17$ & $84.7 \pm 22$ & $29.4 \pm 11$ & $8.9 \pm 2$ & $52.1 \pm 13$ \\
\hline Myopic eyes (luminosity 2) & $67.2 \pm 21$ & $83.6 \pm 13$ & $117.0 \pm 23$ & $52.1 \pm 14$ & $19.1 \pm 3$ & $67.8 \pm 15$ \\
\hline Myopic eyes (luminosity 3) & $71.8 \pm 15$ & $85.1 \pm 14$ & $117.9 \pm 43$ & $64.5 \pm 22$ & $26.0 \pm 12$ & $73.1 \pm 21$ \\
\hline Myopic eyes (luminosity 4) & $67.8 \pm 12$ & $87.8 \pm 15$ & $120.0 \pm 38$ & $70.3 \pm 19$ & $30.4 \pm 8$ & $75.3 \pm 18$ \\
\hline Cataract eyes (luminosity 1 ) & $31.2 \pm 14$ & $30.0 \pm 13$ & $16.1 \pm 8$ & $1.0 \pm 0.1$ & $0.0 \pm 0.0$ & $15.7 \pm 7$ \\
\hline Cataract eyes (luminosity 2) & $35.0 \pm 14$ & $38.7 \pm 16$ & $25.2 \pm 11$ & $7.9 \pm 2$ & $0.8 \pm 0.1$ & $21.5 \pm 8.6$ \\
\hline Cataract eyes (luminosity 3 ) & $42.3 \pm 12$ & $48.9 \pm 21$ & $38.0 \pm 23$ & $11.4 \pm 6$ & $3.2 \pm 1$ & $28.8 \pm 13$ \\
\hline Cataract eyes (luminosity 4) & $41.0 \pm 22$ & $48.9 \pm 23$ & $34.3 \pm 11$ & $14.0 \pm 7$ & $5.1 \pm 2$ & $28.7 \pm 13$ \\
\hline
\end{tabular}

Values are presented as means $\pm \mathrm{SD} .{ }^{\text {a }}$ For healthy eyes.

according to luminosities and optotype frequencies (table 1; fig. 3). There was a 3-sided significant difference between healthy, cataract, and myopic eyes for mean linear contrast sensitivity values depending on the optotype frequency and luminosity ( $\mathrm{p}<0.05$, Kruskal-Wallis test). Two-sided analysis revealed a significant difference between mean contrast sensitivity values for all optotype frequencies as well as all luminosities in healthy versus cataract and myopic versus cataract eyes ( $\mathrm{p}<0.05$, MannWhitney $U$ test). Analysis of differences between myopic and healthy eyes showed slightly decreased mean values of contrast sensitivity, significant only for the lowest luminosity (i.e. 1) and the biggest optotype bars (A1, p < 0.05, Mann-Whitney U test). 

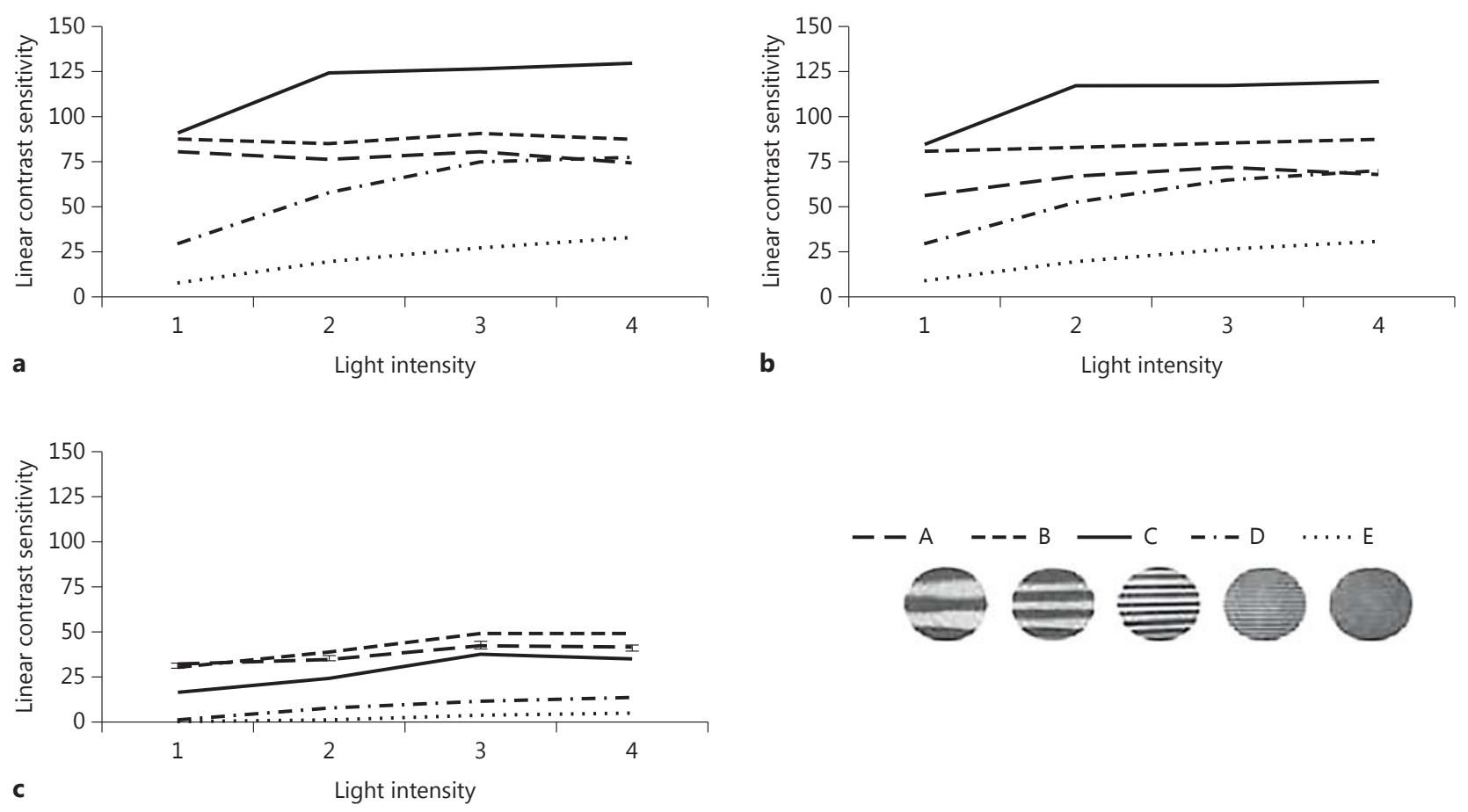

Fig. 4. Contrast sensitivity according to spatial frequency and light intensity for emmetropic (a), myopic (b), and cataract eyes $(\mathbf{c})$.

\section{Differences within Groups}

The optotype with a spatial frequency of $3.0 \mathrm{cpd}$ provided the best (but still not optimal) contrast sensitivity in eyes with cataract, while healthy and myopic eyes reached the best results for optotype with a spatial frequency of $6.0 \mathrm{cpd}$ (fig. $4 \mathrm{a}-\mathrm{c}$ ). The increase in light intensity had a positive effect on the average contrast sensitivities in the examined eyes, except for eyes with cataract, where the maximum light intensity did not improve the contrast sensitivity and worsened it for optotypes with lower frequencies $(1.5,3.0$, and $6.0 \mathrm{cpd}$ ) (fig. 3a, 4c; table 2).

\section{Contrast Vision Defects}

Healthy eyes tended to have slightly better values of contrast sensitivity than myopic ones; however, there was a very small difference between these 2 groups, especially for high spatial frequencies $(12.0$ and $18.0 \mathrm{cpd})$, where the reverse relation was observed for optotype $\mathrm{E}$ (18.0 cpd) with the lowest luminosity, i.e. 1 (the E1 con-

Impact of Luminosity on Contrast Vision

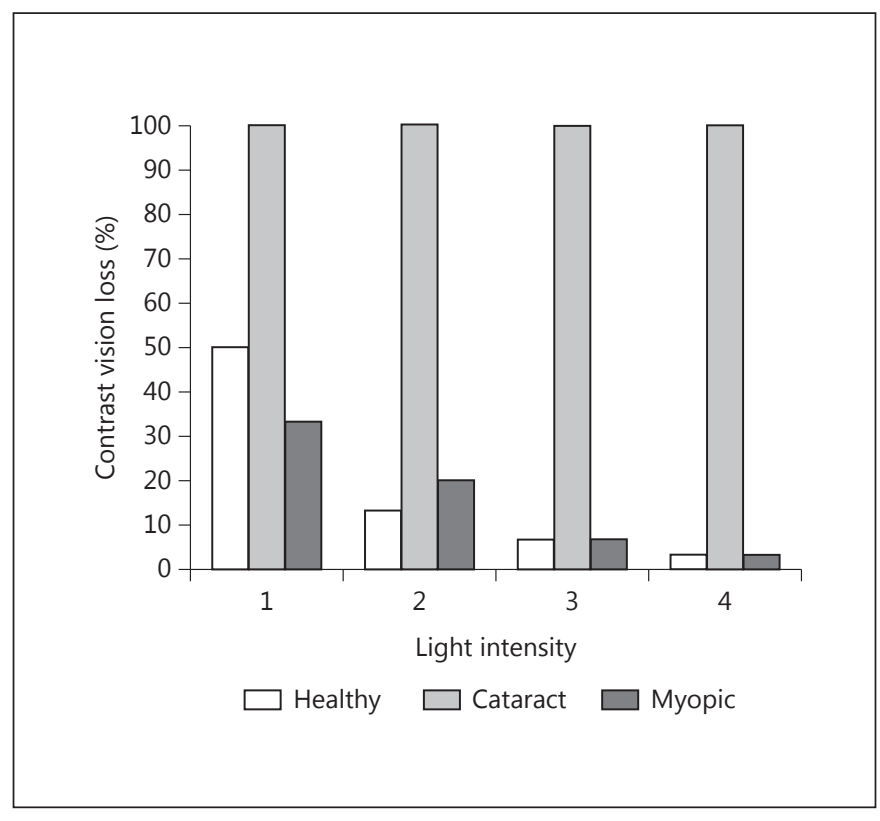

Fig. 5. Contrast vision defects within groups. 
Fig. 6. Luminosity value of the reading sur-

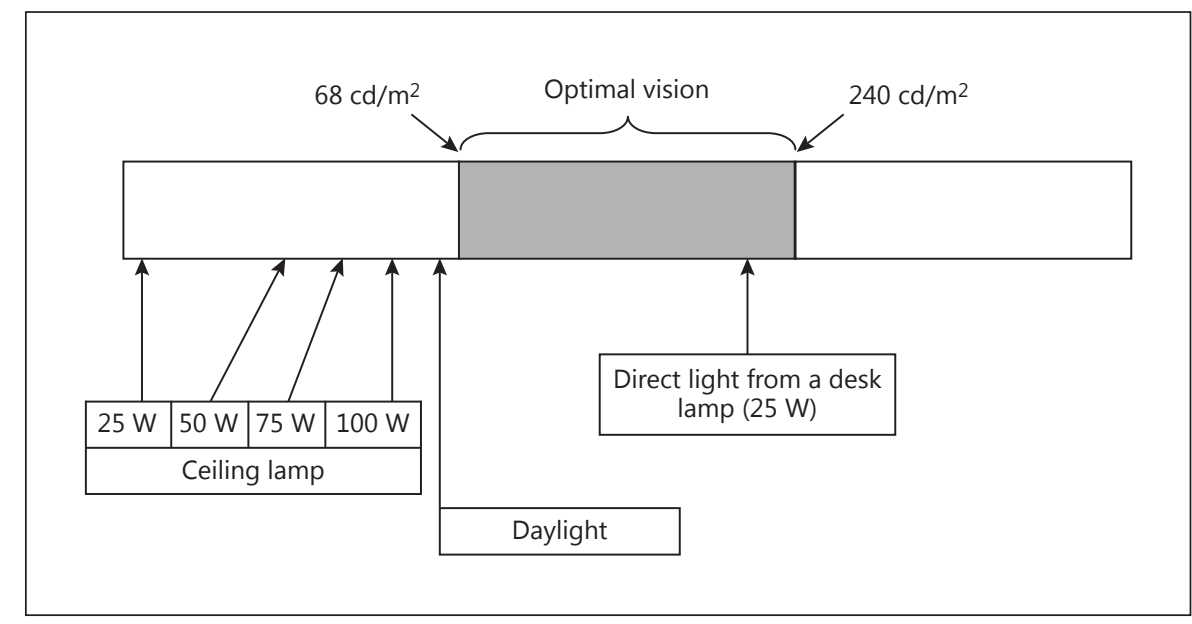
face illuminated by different light sources.

Table 2. Statistical analysis of differences between contrast sensitivity values depending on the applied light intensity within groups of healthy, myopic, and cataract eyes

\begin{tabular}{lccc}
\hline Resolution & \multicolumn{2}{l}{ p values } & \\
\cline { 2 - 4 } & healthy eyes & myopic eyes & cataract eyes \\
\hline A1 vs. A2 & n.s. & n.s. & n.s. \\
A2 vs. A3 & n.s. & n.s. & $<0.05$ \\
A3 vs. A4 & n.s. & n.s. & n.s. \\
B1 vs. B2 & n.s. & n.s. & $<0.05$ \\
B2 vs. B3 & n.s. & n.s. & $<0.05$ \\
B3 vs. B4 & n.s. & n.s. & n.s. \\
C1 vs. C2 & $<0.05$ & $<0.05$ & $<0.05$ \\
C2 vs. C3 & n.s. & n.s. & $<0.05$ \\
C3 vs. C4 & n.s. & n.s. & n.s. \\
D1 vs. D2 & $<0.05$ & $<0.05$ & $<0.05$ \\
D2 vs. D3 & $<0.05$ & $<0.05$ & $<0.05$ \\
D3 vs. D4 & n.s. & n.s. & $<0.05$ \\
E1 vs. E2 & $<0.05$ & $<0.05$ & $<0.05$ \\
E2 vs. E3 & $<0.05$ & $<0.05$ & $<0.05$ \\
E3 vs. E4 & $<0.05$ & n.s. & $<0.05$ \\
\hline
\end{tabular}

A-E describe spatial frequencies, and 1-4 refer to light intensities. $\mathrm{p}<0.05$ was considered statistically significant. n.s. $=$ Not significant.

trast sensitivity for myopic eyes was higher than for healthy ones: $8.9 \pm 2$ and $7.9 \pm 2$, respectively; table 1 ). In healthy and myopic eyes, increased light intensity did not result in contrast sensitivity improvement for $\mathrm{A}(1.5 \mathrm{cpd})$ or B (3.0 cpd) optotype frequencies and showed clear improvement for C-E (6.0-18.0 cpd) optotypes. For cataract eyes, no optimal light intensity was found that could ensure a mean contrast sensitivity within the normal limits. In the single frequency analysis, increased luminosity (i.e. 3) allowed vision to reach the lower normative limit of contrast sensitivity (first row in table 1) for optotypes $A$ and $B$ in cataract eyes; however, further increasing the luminosity (i.e. to 4) did not improve contrast vision. This relation was absent for optotypes C-E (6.0-18.0 cpd), where none of the luminosities improved vision enough to reach optimal contrast sensitivity values (table 1). Contrast vision defects were described as the percentage of out-of-limits results for each light intensity. Even a single result beyond the norm for a single light intensity was classified as a visual defect. In this analysis, all of the examined cataract eyes showed visual defects. For the lowest light intensity, myopic eyes showed fewer deficits than healthy eyes, and this was reversed for higher intensities (fig. 5).

Luminosity, as an Environmental Factor, Depends on Applied Light Sources

Based on our measurements, most of the daily used indoor light sources, including diffuse daylight, do not provide illumination that ensures optimal vision, which ranges between 68 and $240 \mathrm{~cd} / \mathrm{m}^{2}$. For reading purposes, only short-distance, direct illumination of the surface results in optimal vision conditions (fig. 6).

\section{Discussion}

Our study revealed that cataract eyes required a different method to improve their contrast sensitivity than eyes with preserved optic media transparency. The increase in 
light intensity had a positive effect on the average contrast sensitivity in emmetropic and myopic eyes but not in eyes with cataract, where the maximum light intensity did not improve contrast sensitivity and could even worsen it for 3 of the 5 examined spatial frequencies. This observation could be a basis for the practical usefulness of our results. For healthy and myopic eyes, all of the applied luminosities ensured contrast sensitivities within the optimal limits. Large optotypes remained out of the influence of light intensity, but contrast vision for small optotypes was clearly improved after more intense light was used. Based on this, contrast sensitivity and therefore the quality of vision could be improved by applying increased object luminosity (light intensity) for emmetropic and myopic eyes. For eyes with cataracts, the reasoning is different. In contrast to other groups, submaximal luminosity (intensity 3, i.e. $154 \mathrm{~cd} / \mathrm{m}^{2}$ ) worked better than the strongest light, which did not cause vision improvement. Large optotypes (A and B) were characterized by satisfying contrast sensitivities, which together with optimal light intensity yielded values just above the lower limit of normal. For small optotypes, although there was a slight improvement in contrast vision depending on increased light intensity up to the submaximal value (i.e. 3 ), contrast sensitivities remained below the limit for optimal vision. The above course of thinking allows generalization of the fact that, for cataract eyes, the size of observed objects and their contrast ensure a better quality of vision than higher luminosity alone, which can yield the slight improvement observed in examination charts; however, this does not provide good enough visual quality.

It has been reported that visual acuity is an insufficient tool in ophthalmic diagnostics, and contrast sensitivity measurement might serve as an additional diagnostic method in healthy eyes as well as in various ophthalmic diseases [16-18]. Contrast vision depends on many anatomical factors, such as the opacity of the ocular optic media, the functional state of the retina and the optic nerve, and environmental factors, primarily light intensity and the resolution of the observed images [8]. In our study, due to the reduced transparency of the optic media in simulated cataract, contrast sensitivity had the lowest values among all of the studied groups, similar to the results of previous studies $[19,20]$. Changes that were observed in the cataract simulation eyes (decreased contrast sensitivity to mostly high spatial frequencies of optotypes), according to other authors' studies, could be interpreted as cortical cataract equivalent since this type of cataract is a cause of similar cataract vision defects [8]. Although the myopic eyes in our study had slightly worse values of contrast sensitivity than the healthy eyes, the percentage of contrast vision loss for the lowest light intensity was lower than in healthy eyes, similar to previously reported findings [21]. A plausible explanation for this situation could be found if myopia is considered a complex disease that involves not only refractive error but also secondary retinal changes, different pupil sizes, and correction-related amblyopia $[8,22]$.

The findings of this study indicate a need to focus on further analyses of the conditions that should be created to improve the visual quality and comfort of cataract patients. Conditions of submaximal light intensity, which were revealed to be the best for contrast sensitivity improvement, can be created by direct illumination of the reading surface with a 25 -watt light source from a short distance, e.g. a desk lamp (fig. 6). Since cataract is the leading cause of blindness worldwide (though reversible), it is particularly important to ensure that patients have the best quality of vision, not merely the best visual acuity. Contrast sensitivity assessment could be applied in these cases. It could be used not only as a diagnostic instrument but also to help to improve the quality of life of cataract patients and after intraocular lens implantation due to cataract surgery, since simple visual acuity measurement serves only as a quantitative examination.

\section{Conclusions}

The light intensity level had a positive effect on the contrast sensitivity of the examined eyes, except for eyes with cataract simulation, where even the maximum light intensity did not improve contrast vision. This could indicate that patients with cataracts require increased contrast of text rather than brighter illumination to improve their quality of life.

\footnotetext{
References $\longrightarrow 1$ Owsley C: Contrast sensitivity. Ophthalmol Clin North Am 2003;16:171-177.

$\checkmark 2$ Ginsburg AP: Contrast sensitivity and functional vision. Int Ophthalmol Clin 2003;43: 5-15.

3 Amesbury EC, Schallhorn SC: Contrast sensitivity and limits of vision. Int Ophthalmol Clin 2003;43:31-42.

4 Barash S, Melikyan A, Sivakov A, et al: Shift of visual fixation dependent on background illumination. J Neurophysiol 1998;79:27662781.

5 Solomon SG, Lennie P: The machinery of colour vision. Nat Rev Neurosci 2007;8:276286
} 
6 Marc R: Functional neuroanatomy of the retina; in Albert D, Miller J (eds): Albert and Jakobiec's Principles and Practice of Ophthalmology, ed 3. New York, Elsevier, 2008, pp 1565-1592.

7 Rockhill R, Daly F, MacNeil M, et al: The diversity of ganglion cells in a mammalian retina. J Neurosci 2002;22:3831-3843.

8 Hashemi H, Khabazkhoob M, Jafarzadehpur $\mathrm{E}$, et al: Contrast sensitivity evaluation in a population-based study in Shahroud, Iran. Ophthalmology 2012;119:541-546.

9 Plainis S, Anastasakis AG, Tsilimbaris MK: The value of contrast sensitivity in diagnosing central serous chorioretinopathy. Clin Exp Optom 2007;90:296-298.

10 McKendrick AM, Sampson GP, Walland MJ, et al: Contrast sensitivity changes due to glaucoma and normal aging: low-spatial-frequency losses in both magnocellular and parvocellular pathways. Invest Ophthalmol Vis Sci 2007;48:2115-2122.
11 Chylack LT Jr, Padhye N, Khu PM, et al: Loss of contrast sensitivity in diabetic patients with LOCS II classified cataracts. Br J Ophthalmol 1993;77:7-11.

$>12$ Georgakopoulos CD, Eliopoulou MI, Exarchou AM, et al: Decreased contrast sensitivity in children and adolescents with type 1 diabetes mellitus. J Pediatr Ophthalmol Strabismus 2011;48:92-97.

13 Chua BE, Mitchell P, Cumming RG: Effects of cataract type and location on visual function: the Blue Mountains Eye Study. Eye (Lond) 2004; 18:765-772.

14 Montes-Mico R, Alio JL, Munoz G: Contrast sensitivity and spatial-frequency spectrum after refractive surgery (letter). J Cataract Refract Surg 2003;29:1650-1651.

15 Ashworth B, Aspinall PA, Mitchell JD: Visual function in multiple sclerosis. Doc Ophthalmol 1989;73:209-224.

16 Thayaparan K, Crossland MD, Rubin GS: Clinical assessment of two new contrast sensitivity charts. Br J Ophthalmol 2007;91:749752.
17 Haegerstrom-Portnoy G: The Glenn A. Fry Award Lecture 2003: vision in elders - summary of findings of the SKI study. Optom Vis Sci 2005;82:87-93.

18 Hawkins AS, Szlyk JP, Ardickas Z, et al: Comparison of contrast sensitivity, visual acuity, and Humphrey visual field testing in patients with glaucoma. J Glaucoma 2003;12:134-138.

19 Adamsons I, Rubin GS, Vitale S, et al: The effect of early cataracts on glare and contrast sensitivity: a pilot study. Arch Ophthalmol 1992;110:1081-1086.

20 Hong YT, Kim SW, Kim EK, et al: Contrast sensitivity measurement with 2 contrast sensitivity tests in normal eyes and eyes with cataract. J Cataract Refract Surg 2010;36:547552.

21 Liou SW, Chiu CJ: Myopia and contrast sensitivity function. Curr Eye Res 2001;22:81-84.

22 Heng WJ, Oen FT, Peng CM: Effects of optical correction media on contrast sensitivity. Ann Acad Med Singapore 1997;26:18-21. 